\title{
Avaliação do perfil clínico e laboratorial de gatos hipertireoideos com ou sem lobo tireoidiano aumentado a palpação cervical ${ }^{1}$
}

\author{
Vanessa P. de Faria ${ }^{2}$, Katia B. Corgozinho ${ }^{3 *}$, Lio Moreira ${ }^{3}$, Ana M.R. Ferreira ${ }^{3}$ \\ e Heloisa J.M. de Souza²
}

\begin{abstract}
Faria V.P., Corgozinho K.B., Moreira L., Ferreira A.M.R. \& Souza H.J.M. 2013. [Clinical and laboratorial evaluation of hyperthyroid domestic cats with or without palpable thyroid gland.] Avaliação do perfil clínico e laboratorial de gatos hipertireoideos com ou sem lobo tireoidiano aumentado a palpação cervical. Pesquisa Veterinária Brasileira 33(1):80-85. Departamento de Clínica e Cirurgia Animal, Instituto de Veterinária, Universidade Federal Rural do Rio de Janeiro, Seropédica, RJ 23890-000, Brazil. E-mail: katia.barao@gmail.com

Hyperthyroidism is the most common endocrine disorder of older cats. Due to the progressive nature of the disease, the identification of a subclinical stage is essential in order to have a better control of the disease. The present study was a survey of the frequency of feline hyperthyroidism in Rio de Janeiro, from March, 2007 to April, 2008. The purpose of this study was to perform an evaluation of the clinical, laboratorial and echocardiographic aspects in a feline population of naturally acquired hyperthyroidism, with or without palpable thyroid gland. The selection of the cats was done by the documentation of an elevation of total thyroxine (TT4) levels by radioimmunoassay. The animals were divided in two groups according to the thyroid palpation: group I (non-palpable thyroid lobe) and group II (palpable thyroid lobe). Laboratorial and complete clinical evaluations were performed in all cats. The clinical evaluation included behavioral evaluation, body weight, body condition, dermatologic, cardiopulmonary auscultation, thyroid palpation and systolic blood pressure reading. Besides the TT4, the following exams were performed: urea, creatinine, alanine aminotransferase (ALT), alkaline phosphatase (ALP) and glucose. Thirty one cats had an echocardiogram performed. On the basis of TT4 concentrations, 51 of the 178 cats were classified as hyperthyroid. One or more enlarged thyroid glands were palpated in 25 of the 51 hyperthyroid cats and were included at Group II. Twenty six cats didn't palpable thyroid gland and were included at Group I. Clinical, laboratorial and echocardiographic parameters were similar between the two groups. However, the TT4 levels were significantly lower in the non-palpable thyroid group. All geriatric cats must be thyroid gland palpable on clinic examination and have TT4 measured. Many hyperthyroid cats may be asymptomatic and early diagnosis prevents organ damage such as liver, kidneys and heart.
\end{abstract}

INDEX TERMS: Feline, hyperthyroidism, thyroid palpation, total thyroxine.

RESUMO.- 0 hipertireoidismo é a endocrinopatia mais comum em gatos idosos. Devido à natureza progressiva da doença, a identificação de um estágio subclínico se torna

\footnotetext{
${ }^{1}$ Recebido em 10 de julho de 2012.

Aceito para publicação em 5 de novembro de 2012.

${ }^{2}$ Departamento de Medicina e Cirurgia Veterinária, Instituto de Veterinária, Universidade Federal Rural do Rio de Janeiro (UFRRJ), BR465 Km 7, Seropédica, RJ 23890-000, Brasil.

${ }^{3}$ Departamento de Clínica e Reprodução Animal, Faculdade de Veterinária, Universidade Federal Fluminense (UFF), Rua Vital Brasil Filho 64, Niterói, RJ 24.230-340. *Autor para correspondência: katia.barao@gmail.com
}

essencial para melhor controle da doença. 0 presente estudo compreendeu um levantamento da frequência de gatos hipertireoideos durante o período de março de 2007 a abril de 2008, no estado do Rio de Janeiro. 0 objetivo deste trabalho foi avaliar os aspectos clínicos, laboratoriais e ecocardiográficos em uma população de gatos domésticos com hipertireoidismo naturalmente adquirido, com ou sem lobo tireoidiano palpável. A seleção dos animais foi realizada através da detecção do valor de tiroxina $\left(\mathrm{T}_{4}\right)$ total acima dos valores de referência com a técnica de radioimunoensaio. Os animais foram divididos em dois grupos com 
base na identificação do aumento da tireoide à palpação: Grupo I (tireoide não palpável) e Grupo II (tireoide palpável). Avaliações clínica e laboratoriais completas foram realizadas em todos os gatos do estudo. A avaliação clínica incluiu avaliação comportamental, peso, escore de condição corporal, pele e pelagem, auscultação cardiopulmonar, palpação tireoidiana e aferição da pressão arterial sistólica. Além do $\mathrm{T}_{4}$ total, os seguintes exames laboratoriais foram realizados: hemograma, ureia, creatinina, alanina aminotransferase (ALT), fosfatase alcalina (FA) e glicose. Trinta e um animais foram submetidos à avaliação cardíaca, através de ecocardiograma. Dos 178 animais avaliados, 51 foram incluídos nesse estudo por apresentarem aumento sérico da $\mathrm{T}_{4}$. Vinte e seis animais foram inclusos no Grupo I e vinte e cinco no Grupo II. Os parâmetros clínicos, laboratoriais e ecocardiográficos foram muito semelhantes entre os dois grupos. No entanto, os níveis séricos de $\mathrm{T}_{4}$ total foram significativamente menores nos gatos com tireoide não palpável. Todo gato acima de sete anos deve ser avaliado para presença de hipertireoidismo através da palpação tireoidiana e avaliação sérica de tiroxina total, pois muitos gatos sem sinais clínicos apresentam hipertireoidismo e o diagnóstico precoce permite diminuir os efeitos sistêmicos da doença em órgãos como fígado, rins e coração.

TERMOS DE INDEXAÇÃO: Felino, hipertireoidismo, lobo tireoidiano, tiroxina total.

\section{INTRODUÇÃO}

O hipertireoidismo é uma doença endócrina comum em felinos que acomete machos e fêmeas, principalmente acima de sete anos de idade, e não tem predisposição racial (Naan et al. 2006, Júnior et al. 2007, Souza et al. 2007, Harvey et al. 2009). É uma síndrome clínica que resulta da circulação excessiva dos hormônios ativos da tireoide, triiodotironina $\left(\mathrm{T}_{3}\right)$ e tiroxina $\left(\mathrm{T}_{4}\right)$ total, produzidos por uma glândula anormalmente funcionante e tem se tornado uma enfermidade extremamente importante e comum em gatos idosos (Naan et al. 2006, Peterson \& Ward 2007).

Os sinais clínicos são insidiosamente progressivos e o proprietário pode inicialmente ignorá-los ou interpretá-los apenas como indícios de que seu gato está ficando mais velho (Meric 1989). Os sinais clínicos clássicos de hipertireoidismo incluem hiperatividade, perda de peso, polifagia, poliúria, polidipsia, diarreia, fraqueza muscular, aumento de volume fecal, padrão respiratório ofegante, vômito, crescimento rápido das unhas, alopecia e flexão ventral de pescoço (Broussard et al. 1995, Feldman \& Nelson 2004, Peterson 2006).

O diagnóstico é baseado no histórico, presença de lobos tireoidianos cervicais palpáveis e confirmado através do exame hormonal dosando a tiroxina sérica total (Feldman \& Nelson 2004, Júnior et al. 2007, Paepe et al. 2008).

0 hipertireoidismo causa desordens múltiplas, aumentando a taxa metabólica e o consumo de oxigênio, e reduzindo a resistência vascular periférica (Fox et al. 1999). Por isso, além da avaliação da função da glândula tireoide em gatos com suspeita de hipertireoidismo, é de extrema importância a avaliação precisa de outros sistemas para identificar doenças concomitantes, e também, para descartar desordens que simulem o hipertireoidismo, tais como diabetes mellitus, insuficiência renal, cardiopatias, hepatopatias, má digestão, má absorção e neoplasias (Broussard 1995, Feldman \& Nelson 2004).

A precocidade no diagnóstico em animais idosos tem os seguintes objetivos: 1) promover longevidade e melhorar a qualidade de vida dos pacientes felinos idosos através do reconhecimento e controle de fatores de risco, 2) detectar doenças na fase pré-clínica, 3) corrigir ou retardar a progressão de desordens já existentes e 4) melhorar ou manter as funções remanescentes. Deve-se preconizar um programa de saúde preventivo para gatos acima de onze anos de idade, incluindo testes diagnósticos ao menos anuais de hemograma completo, creatinina sérica, alanina aminotransferase (ALT), fosfatase alcalina (FA) e $\mathrm{T}_{4}$ total por radioimunoensaio (Richards et al. 2005).

O objetivo desse trabalho é descrever a ocorrência de hipertireoidismo em gatos geriátricos no período de dois anos e avaliar o perfil clínico e laboratorial dos animais hipertireoideos com glândulas tireoidianas palpáveis e não palpáveis.

\section{MATERIAL E MÉTODOS}

Foram atendidos 178 gatos para avaliação clínica geriátrica de rotina ou pacientes acima de sete anos sintomáticos com queixas clínicas diversas no período de 2007 a 2008. Como critérios para inclusão dos animais foram estabelecidos os valores de $\mathrm{T}_{4}$ total acima de 29,9ng/mL (referência 15-30ng/mL, Bet laboratório, Rio de Janeiro, RJ), sem tratamento prévio para hipertireoidismo e idade superior a sete anos, conforme proposto pela Associação Americana de Praticantes da Medicina Felina/Academia de Medicina Felina que recomenda a avaliação da glândula tireoide a partir dos sete anos de idade (Richards et al. 2005).

Os animais com aumento dos níveis séricos de $\mathrm{T}_{4}$ total, foram divididos em dois grupos com base na identificação de aumento da tireoide à palpação, o Grupo I incluiu os animais sem glândula palpável e o Grupo II incluiu animais com aumento cervical evidente da glândula tireoide. A pesquisa seguiu as normas descritas pelo Colégio Brasileiro de Experimentação Animal (COBEA).

Os dados de todos os animais foram registrados em fichas individuais incluindo a resenha, histórico e anamnese. 0 exame clínico seguiu os métodos semiológicos de rotina, incluindo avaliação clínica detalhada. Durante a avaliação clínica foram observadas:

1) Características comportamentais: se o animal é hiperativo, inquieto (difícil manipulação), agressivo (aqueles que atacavam) ou calmo.

2) Registro do peso corporal: avaliação do escore de condição corporal (ECC), variando de "1" (muito magro) a "5" (obeso) (Edney \& Smith 1986).

3) Frequência respiratória (FR): movimentos de expansão da cavidade torácica no período de um minuto, normal entre 20 e 30 movimentos por minuto (mpm) (Gonçalves 2004).

4) Pelagem: alterações de coloração, textura, oleosidade e homogeneidade do pelame.

5) Ausculta cardíaca: avaliação de ritmo, presença ou ausência de sopro e da frequência cardíaca (FC), normal entre 140 a 240 batimentos por minuto (bpm) (Camacho \& Mucha 2004).

6) Palpação tireoidiana: a palpação da região cervical foi realizada sempre pelo mesmo profissional para se obter maior acurácia uma vez que se trata de técnica subjetiva e semi-quantitativa. A técnica escolhida foi a descrita por Norsworthy et al. 2002b. Se o lobo tireoidiano não era percebido na primeira palpação, duas no- 
vas palpações eram realizadas após reposicionamento da cabeça do animal. A palpação foi classificada utilizando uma escala de 0 a 6 onde "0" correspondia a lobo não palpável, "1 1 "lobo de tamanho aproximado de 0,5 cm, " 2 " lobo de tamanho aproximado de 0,75cm, "3" lobo de tamanho aproximado de 1,0cm, "4" lobo de tamanho aproximado de $1,5 \mathrm{~cm}$, "5 " lobo de tamanho aproximado de 2,0cm e "6" lobo de tamanho aproximado de maior ou igual a 2,5cm (Norsworthy et al. 2002b).

7) Pressão arterial sistólica (PAS): As mensurações foram realizadas no domicílio dos animais seguindo recomendação de Syme (2007) em relação à ocorrência de estresse. Todos os valores foram comparados com o padrão de referência para um gato normal alerta (referência: 100-160mmHg) (Tilley 2006, Stepien 2011). A pressão arterial sistólica foi mensurada, pelo método não invasivo, ou indireto, com o emprego de um Doppler ultrassônico vascular da marca Minidop ${ }^{\circledR}$ ES-100VX, esfignomanômetro Speidel \& Keller ${ }^{\circledR}$, gel para condução e manguito neonatal descartável $\mathrm{CE}^{\circledR}$ sem que fosse realizada qualquer manipulação prévia. Para mensuração da PAS, o animal foi acomodado em uma mesa ou no colo do proprietário e posicionado em decúbito lateral direito. Em todos os animais foi utilizado o membro pélvico esquerdo. Foram realizadas mensurações com intervalos de 30 segundos para obter a leitura de cinco valores consecutivos da PAS. Fones de ouvido foram utilizados e a PAS final calculada pela média aritmética das cinco aferições. Todas as aferições foram realizadas pelo mesmo operador.

A análise laboratorial incluiu hemograma, ureia, creatinina, fosfatase alcalina, alanina aminotransferase, glicemia e $\mathrm{T}_{4}$ total. Trinta e um animais foram submetidos à avaliação cardíaca, através do ecocardiograma na modalidade M-mode visando identificar cardiomiopatia concomitante (Henik 2002)

Para análise estatística, o teste não paramétrico de Mann-Whitney foi utilizado, adotando o nível de significância de 5\% considerado para todas as análises. Quando a aproximação à normalidade foi considerada apropriada, o teste foi complementado com um teste-t.

\section{RESULTADOS}

Dos 178 animais avaliados, somente 51 animais (29\%) foram caracterizados hipertireoideos com base no valor do $\mathrm{T}_{4}$ total. Vinte e seis animais não apresentaram qualquer alteração identificável à palpação cervical, e foram incluídos no Grupo I. Os 25 animais com aumento cervical evidente da glândula tireoide foram agrupados e denominados Grupo II.

A média de idade dos 51 felinos foi de 13,3 $\pm 3,7$ anos, sendo que 13 animais tinham idade variando entre sete e dez anos e os outros 38 apresentavam idade superior a dez anos. Dentre as 24 fêmeas (47\%) e 27 machos (53\%), apenas dois machos e duas fêmeas não eram castrados. A maioria dos animais (67\%) não tinha raça definida, os outros 17 animais eram das raças Persa (13\%) e Siamês (20\%).

Quanto ao histórico, polifagia foi observada em 8\% do total de animais, enquanto $16 \%$ dos proprietários relataram apetite reduzido. Um dos animais com apetite reduzido apresentava nefropatia crônica concomitante. A perda de peso foi observada em $27 \%$ dos animais. Polidipsia e poliúria foram relatadas em $28 \%$ e $22 \%$ dos animais, respectivamente. Com relação ao funcionamento do trato gastrintestinal, o vômito foi relatado em 14 animais (28\%) e a regurgitação esteve presente em $42 \%$ dos gatos deste grupo.
Quanto ao comportamento na residência, nenhum animal foi descrito como hiperativo ou agressivo, $24 \%$ dos animais foram classificados como inquietos e 76\% como calmos. Vocalização foi relatada pelos proprietários de nove (18\%) animais.

Quanto ao temperamento no consultório, nenhum dos 51 animais apresentou hiperatividade e os demais comportamentos foram observados nas seguintes proporções: inquietação (28\%), agressividade (2\%) e calmos (70\%). A vocalização não foi observada em nenhum animal durante a consulta. O Grupo I e o Grupo II tiveram comportamentos semelhantes.

0 peso dos 51 animais variou de 1,9 a 8,3 $\mathrm{kg}(4,5 \pm 1,6)$. Quanto ao escore de condição corporal (ECC), não houve animal com ECC igual a "1" (muito magro), 33\% (17) apresentaram-se com ECC de "2" (abaixo do peso), a maioria dos animais (51\%) apresentou ECC de "3" (ideal) e oito animais (16\%) enquadraram-se nas classificações acima do peso (ECC de '"4"') e obeso (ECC 5). Não houve diferença entre o escore corporal $(\mathrm{p}=0,21)$ e o peso $(\mathrm{p}=0,83)$ comparando o Grupo I e II.

A média da frequência respiratória foi de 48,40 27,17 mpm e esteve acima dos valores de referência em $84 \%$ dos 51 animais. A avaliação da frequência respiratória mostrou um resultado similar para os dois grupos $(\mathrm{p}=0,52)$.

Dentre os 51 animais, cinco (10\%) apresentaram alterações dermatológicas, sendo dois (4\%) com seborreia seca, um (2\%) com alopecia em dorso, um (2\%) com alopecia auto-induzida e hipotricose, e um (2\%) apenas com hipotricose.

Vinte e sete animais (53\% ou 27/51) apresentaram alterações em auscultação cardiopulmonar. Três animais (6\%) apresentaram os seguintes distúrbios de ritmo: arritmia (2\%) e taquicardia (4\%). O sopro cardíaco foi observado em somente 13 animais (26\%). O sopro variou entre os graus I-III/VI em sete (14\%) dos animais deste estudo, enquanto os outros seis animais com sopro apresentaram grau do sopro superior a III/VI. A frequência cardíaca variou de 125 a 310 bpm, com uma média de 196,0 $\pm 34,48$. Não houve diferença estatística entre os grupos em relação a frequência cardíaca $(p=0,72)$ e a presença de sopro cardíaco $(p=0,15)$

0 aumento tireoidiano cervical unilateral foi mais frequente (64\%, 16/25 animais), sendo que a maioria apresentava a tireoide esquerda aumentada (13/16 animais). 0 comprometimento bilateral foi observado em somente $36 \%$ dos animais. Onze dos 25 animais (44\%) apresentaram discreto aumento tireoidiano, onde a(s) glândula(s) não ultrapassava(m) o comprimento de 1,5 cm ("3"). Os 14 animais (56\%) restantes apresentaram glândulas classificadas entre os graus " 4 "e " 6 ". Foi observado relação entre o aumento das tireoides e o aumento do $\mathrm{T}_{4}$ total.

A pressão arterial sistólica variou de 110 a $240 \mathrm{mmHg}$ $(160,0 \pm 32,04)$. No grupo I, doze animais (46\%) apresentaram valores acima dos limites de referência, caracterizando um quadro de hipertensão arterial sistêmica, enquanto que no Grupo II, a hipertensão arterial sistêmica foi observada em sete animais deste grupo (28\%). Apesar disso, as diferenças entre grupos não foram estatisticamente significativas $(p=0,84)$. 
Somente $10 \%(5 / 51)$ dos animais apresentaram aumento do hematócrito. A média do hematócrito foi de $39,2 \%( \pm 5,6)$ (Quadro 1). 0 número de hemácias variou entre 4,5 e $10,7\left(\mathrm{x} 10^{6} / \mu \mathrm{l}\right)(7,6 \pm 1,25)$ e o valor da hemoglobina variou entre 8 e $15,9 \mathrm{~g} / \mathrm{dL}(12,6 \pm 1,82)$ e apenas $2 \%$ e $8 \%$ dos animais estiveram acima dos valores de referência, respectivamente. Somente um animal (2\%) apresentou leucocitose. Por outro lado, leucopenia foi mais frequente, ocorrendo em 17 (33\%) animais. No entanto, a linfopenia foi significativa $(72 \%)$.

O perfil renal composto por ureia e creatinina apresentou, respectivamente, alterações nas seguintes proporções: $84 \%$ $(43 / 51)$ e $14 \%(7 / 51)$. 0 valor médio das taxas de ureia e creatinina foram $64,1 \mathrm{mg} / \mathrm{dL}( \pm 35,4)$ e $1,8 \mathrm{mg} / \mathrm{dL}( \pm 1,1)$, respectivamente. 0 aumento da ureia foi estatisticamente significante e a creatinina pareceu estar negativamente correlacionada com o aumento do $\mathrm{T}_{4}$ total, porém, em uma relação não muito forte, o que pode ser apenas um efeito da amostra pequena. No entanto, o resultado é estatística $(p=0,009)$ e biologicamente significante, indicando que o aumento de uma unidade no $\mathrm{T}_{4}$ total está associado com uma redução de 0,008 unidades de creatinina (equação da regressão: Creatinina $=2,27-0,008 \mathrm{~T}_{4}$ total, $\mathrm{r}^{2}=8,9 \%$, e $\mathrm{r}^{2}$ ajustado $=7,0 \%$ ).

Vinte e oito animais $(61 \%$ ou $28 / 46)$ apresentaram aumento na atividade sérica de ALT e sete $(19 \%$ ou $7 / 36)$ em FA, cujos valores médios foram 119,8 IU/L $( \pm 107,0)$ e 62,1 IU/L $( \pm 68,3)$ respectivamente. Além da média de ALT entre os animais observados estar significantemente acima do intervalo de referência, há uma clara correlação positiva com o $\mathrm{T}_{4}$ total, ou seja, 1,25 unidades a mais no ALT para cada unidade a mais no $\mathrm{T}_{4}$ total (valor $-\mathrm{p}=0,002, \mathrm{r}^{2}=20 \%$, e $r^{2}$ ajustado=18,2\%). A fosfatase alcalina também apresentou correlação - quanto maior o $\mathrm{T}_{4}$ total, maior a FA. 0 aumento é de cerca de 1,19 para cada unidade de $\mathrm{T}_{4}$ total aumentada (valor $-\mathrm{p}<0,001, \mathrm{r}^{2}=47,5 \%$, e $\mathrm{r}^{2} \mathrm{ajustado}=46 \%$ ).

Por definição das amostras deste estudo, todos os animais tiveram valores de $\mathrm{T}_{4}$ total acima dos valores de referência. No entanto, somente três pacientes $(3 / 51 \mathrm{ou}$ $5,88 \%$ ) apresentaram $\mathrm{T}_{4}$ total maior que $120,0 \mathrm{~g} / \mathrm{mL}$ (referência: $15,0-29,9 \mathrm{ng} / \mathrm{mL}$ ). A dosagem sérica do $\mathrm{T}_{4}$ total variou de 30,0 a 150,0ng/mL, com uma média de 62,2ng/
$\mathrm{mL}( \pm 38,5)$. Mais da metade dos animais $(26 / 51$ ou $51 \%)$ apresentou apenas discreto aumento do valor de $\mathrm{T}_{4}$ total $(30,0-60,0 \mathrm{ng} / \mathrm{mL})$.

A glicemia esteve aumentada em apenas um animal (2\%) do estudo e pareceu haver uma correlação negativa com o valor de $\mathrm{T}_{4}\left(\mathrm{p}=0,052, \mathrm{r}^{2}=6,7 \%\right.$, e $\mathrm{r}^{2}$ ajustado $\left.=4,8 \%\right)$, porém a amostra do estudo é muito pequena pra ter a confiança de 95\% necessária.

Analisando-se os exames hematológicos obtidos nos grupos I (tireoide não palpável) e II (tireoide palpável), nenhuma diferença estatisticamente significante foi encontrada entre os grupos para hematócrito $(\mathrm{p}=0,91)$, para leucometria global $(p=0,47)$, para hematimetria $(p=0,96)$ e para hemoglobina $(\mathrm{p}=0,96)$.

Considerando os exames bioquímicos, apenas a diferença entre a concentração sérica de fosfatase alcalina mostrou-se estatisticamente significante no Grupo II apresentando valores médios consideravelmente maiores $(p=0,03)$. A diferença entre os valores séricos de ureia e creatinina; entretanto, está próxima o suficiente do nível de significância de 5\% para se suspeitar que, com uma maior amostragem, talvez fosse possível evidenciar uma significativa diminuição no Grupo II.

Comparando os níveis de $\mathrm{T}_{4}$ total sérico, os animais do Grupo II apresentaram níveis de $\mathrm{T}_{4}$ notadamente superiores aos animais do Grupo I ( $p=0,005)$ e exibiram relação entre o tamanho das glândulas tireoidianas e o $\mathrm{T}_{4}$ total (Fig.1).

0 ecocardiograma foi realizado em $61 \%$ dos animais (31/51) do estudo. A anormalidade mais comumente observada em $74 \%$ dos gatos foi hipertrofia do septo interventricular durante a diástole, variando de 0,3 a $0,9 \mathrm{~cm}$ $(0,5 \pm 0,11)$. Nenhuma diferença estatisticamente significante foi encontrada entre os animais do Grupo I e do Grupo II.

\section{DISCUSSÃO}

A descrição de casos de hipertireoidismo no Brasil ainda é escassa. Esta série de gatos hipertireoideos é a primeira largamente descrita em detalhes e com um significativo número de animais, dentro de um curto período de tempo, em nosso país. 0 primeiro relato em nível nacional ocorreu somente no ano de 2005 (Carlos \& Albuquerque 2005).

Quadro 1. Exames realizados nos 51 gatos domésticos mostrando a média, desvio padrão, valores mínimo, máximo e número de animais acima e abaixo dos valores de referência

\begin{tabular}{|c|c|c|c|c|c|c|}
\hline \multirow[t]{2}{*}{ Variável } & \multirow[t]{2}{*}{$\mathrm{N}$} & \multirow{2}{*}{ Média \pm DP } & \multirow{2}{*}{$\begin{array}{c}\text { Valor } \\
\text { mínimo }\end{array}$} & \multirow{2}{*}{$\begin{array}{c}\text { Valor } \\
\text { máximo }\end{array}$} & \multicolumn{2}{|c|}{ Número de animais } \\
\hline & & & & & $\begin{array}{l}\text { Acima dos valo- } \\
\text { res de referência }\end{array}$ & $\begin{array}{l}\text { Abaixo dos valo- } \\
\text { res de referência }\end{array}$ \\
\hline \multicolumn{7}{|l|}{ Dosagem hormonal } \\
\hline Tiroxina sérica total $(\mathrm{ng} / \mathrm{mL})$ & 51 & $62,2 \pm 38,52$ & 30,0 & 150,0 & $51(100 \%)$ & - \\
\hline \multicolumn{7}{|l|}{ Bioquímica sérica } \\
\hline Ureia $(\mathrm{mg} / \mathrm{dL})$ & 51 & $63,5 \pm 35,28$ & 34,0 & 260,0 & $19(37 \%)$ & 0 \\
\hline Creatinina $(\mathrm{mg} / \mathrm{dL})$ & 51 & $1,7 \pm 1,09$ & 0,7 & 7,5 & $10(20 \%)$ & 0 \\
\hline Alanina aminotransferase (IU/L) & 46 & $119,8 \pm 107,09$ & 915,0 & 412,0 & $28(61 \%)$ & 0 \\
\hline Fosfatase alcalina (IU/L) & 36 & $62,1 \pm 68,31$ & 0,0 & 323,0 & $7(19 \%)$ & 0 \\
\hline Glicose $(\mathrm{mg} / \mathrm{dL})$ & 51 & $94 \pm 22,87$ & 60 & 177 & $1(2 \%)$ & 0 \\
\hline \multicolumn{7}{|l|}{ Hemograma } \\
\hline Hematócrito (\%) & 51 & $39,2 \pm 5,62$ & 24 & 48,3 & $5(10 \%)$ & 0 \\
\hline Hemácias $\left(\mathrm{x} 10^{6} / \mu \mathrm{L}\right)$ & 51 & $7,6 \pm 1,25$ & 4,5 & 10,7 & $1(2 \%)$ & $2(4 \%)$ \\
\hline Hemoglobina $(\mathrm{g} / \mathrm{dL})$ & 51 & $12,6 \pm 1,82$ & 8 & 15,9 & $4(8 \%)$ & 0 \\
\hline Leucometria global $\left(\times 10^{3} / \mu \mathrm{lL}\right)$ & 51 & $7,58 \pm 3,6807$ & 2,3 & 20,5 & $1(2 \%)$ & $17(34 \%)$ \\
\hline
\end{tabular}




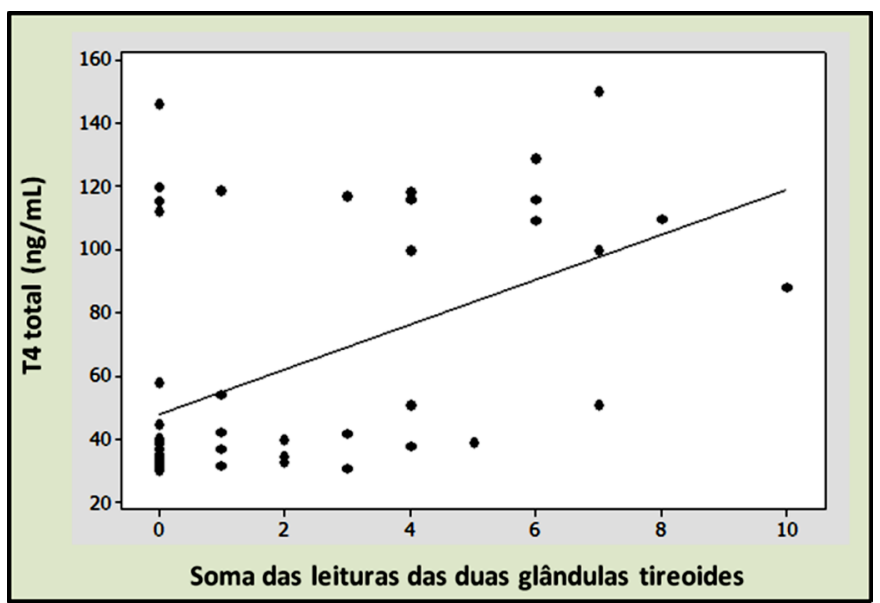

Fig.1. Comparação do valor de $\mathrm{T}$ total com o tamanho das glândulas tireoidianas (IC >3,59 e <10,61).

Posteriormente, Souza et al. (2007) relataram 43 casos de felinos hipertireoideos atendidos em um período de 7 anos (1999-2006) e Júnior et al. (2007) relataram somente 8 casos em um período de 4 anos (2000-2003). Sugere-se que o hipertireoidismo não deva ser uma doença nova e, simplesmente, vem sendo diagnosticada mais frequentemente uma vez que gatos têm vivido mais e proprietários têm tido maior cuidado com os pacientes geriátricos (Kass et al. 1999). Os sinais clínicos e alterações observados nos animais do presente estudo foram discretos quando comparados aos apresentados pelos trabalhos anteriormente mencionados, provavelmente porque os gatos foram trazidos para avaliação de rotina e não com a sinais clínicos de hipertireoidismo, tendo um diagnóstico precoce da doença.

0 animal hipertireoideo geralmente é magro e em cerca de $95 \%$ dos casos apresenta idade igual ou superior a 10 anos no momento do diagnóstico (Broussard et al. 1995, Naan et al. 2006, Peterson 2006). A média de idade dos gatos diagnosticados com hipertireoidismo no presente trabalho foi bastante similar ao observado na literatura consultada (Broussard et al. 1995, Norsworthy et al. 2002b, Naan et al. 2006, Souza et al. 2007). Não houve predileção sexual, o que está de acordo com o citado por Naan et al. (2006) e Harvey et al. (2009); entretanto, foi observado, de maneira subjetiva, maior frequência da doença em gatos sem raça definida, provavelmente pelo fato de representarem a maioria nos consultórios em nosso país, conforme anteriormente enfatizado por Júnior et al. (2007) e Souza et al. (2007).

A frequência da hiporexia foi semelhante ao relatado por Peterson et al. (1983), que observaram em seu estudo que períodos de apetite reduzido podem alternar com períodos de apetite normal ou aumentado em gatos hipertireoideos. 0 hipertireoidismo apático não foi observado neste estudo apesar de ser relatado em $10 \%$ dos animais afetados por esta enfermidade (Feldman \& Nelson 2004). No entanto, vale ressaltar o fato de que alguns desses animais com hiporexia apresentavam doenças concomitantes como insuficiência renal crônica e doença intestinal inflamatória, contribuindo também para uma possível explicação para redução do apetite desses pacientes, o que foi relatado também por Peterson (2006).
Apesar de ser considerado um dos sinais clínicos mais comuns, a polifagia foi a alteração menos observada, o que parece estar relacionado com a identificação da doença em sua fase inicial, o que tem ocorrido mais frequentemente com o passar dos anos (Boussard et al. 1995). No entanto, mesmo em estudos recentes (Feldman \& Nelson 2004, Souza et al. 2007), a frequência da polifagia foi significativamente mais expressiva que a aqui observada. 0 fato de a maior parte dos animais apresentarem hipertireoidismo leve, com apenas discreto aumento do hormônio tireoidiano, pode justificar a baixa incidência de sinais clínicos considerados clássicos como vômito, polifagia, polidipsia e poliúria (Peterson 2006).

A maioria dos parâmetros avaliados (clínicos, laboratoriais e ecocardiográficos) não foi significantemente diferente dos relatados em outros estudos devido ao fato de que gatos diagnosticados precocemente apresentam sinais clínicos e alterações laboratoriais e de imagem discretos e, muitas vezes, ainda não evidentes para o proprietário (Broussard et al. 1995, Syme 2007).

Dentre as possíveis anormalidades em bioquímica sanguínea, a atividade elevada das enzimas hepáticas continua sendo a alteração mais comumente associada com esta enfermidade, como observado na clara correlação positiva entre ALT/FA e $\mathrm{T}_{4}$ total. No entanto, como observado neste grupo de 51 animais, o grau de elevação dessas enzimas, quando presente, é mais sutil, por serem casos de hipertireoidismo precoce. Demais alterações relatadas em literatura encontraram-se variavelmente associadas com o hipertireoidismo, oferecendo pouca contribuição para o diagnóstico da doença (Shiel \& Mooney 2007). A fosfatase alcalina significativamente mais elevada em gatos do grupo II pode ser considerada efeito da relação direta entre $T_{4}$ total e tamanho de lobo tireoidiano e, consequentemente, entre lobo tireoidiano e FA.

Devido ao aumento da taxa de filtração glomerular causado pelo estado de tireotoxicose, apenas cerca de $10 \%$ dos animais hipertireoideos são azotêmicos, o que justifica a correlação negativa observada entre creatinina e $\mathrm{T}_{4}$ total (Feldman \& Nelson 2004, Shiel \& Mooney 2007). A maior parte da população estudada apresentou aumento do valor de ureia, o que pode ser esperado devido ao aumento do catabolismo protéico assim como devido à azotemia pré-renal causada pela tireotoxicose (Feldman \& Nelson 2004).

Os lobos tireoidianos geralmente são palpáveis durante a inspeção cervical de gatos hipertireoideos (Junior et al. 2007, Paepe et al. 2008). Acredita-se que, devido ao estágio precoce da doença nos animais deste estudo, muitos ainda não apresentavam hipertrofia da glândula (Norsworthy et al. 2002a). Ainda assim, quase metade do total de animais apresentou tireoide palpável, conforme também descrito por Souza et al. (2007). Isso confirma o fato de que a ausência de lobo tireoidiano palpável não descarta a doença uma vez que tecido tireoidiano ectópico pode estar localizado no mediastino cranial (Harvey et al. 2009, Hibbert et al. 2009). Da mesma maneira que Norsworthy et al. (2002b), a maior parte deste grupo de animais apresentou ao menos uma das glândulas com escore maior ou igual a "4". Portanto, a palpação cervical pode ser forte adjuvante no diagnós- 
tico do hipertireoidismo uma vez que é não invasiva e não tem custo (Paepe et al. 2008). A correlação entre o aumento das tireoides e o aumento do $\mathrm{T}_{4}$ total foi também observada por Norsworthy et al. (2002a) e Boretti et al. (2009), corroborando com a maior probabilidade de hipertireoidismo conforme aumenta o tamanho da glândula tireoidiana.

Em gatos hipertireoideos clássicos, é observado, com maior frequência, aumento tireoidiano bilateral durante o exame físico dos animais (Broussard et al. 1995, Souza et al. 2007). No entanto, no presente estudo, o aumento unilateral foi o mais frequentemente identificado. Norsworthy et al. (2002a) sugeriram que lobos tireodianos não funcionais podem se tornar funcionais e aumentarem de volume. Diante do exposto e do diagnóstico precoce observado nos animais deste estudo, acredita-se que o lobo contralateral poderia progredir quanto ao tamanho, tornando a alteração bilateral quando então identificada em um gato classicamente afetado pela enfermidade.

O hipertireoidismo é frequentemente mencionado como uma importante causa de hipertensão arterial sistêmica em gatos (Richards et al. 2005, Syme 2007). Por esta razão, a pressão arterial sistólica foi avaliada em todos os animais do presente trabalho, enquanto foi avaliada em apenas $19 \%$ dos animais em um dos três estudos anteriormente relatados no Brasil (Souza et al. 2007). A hipertensão arterial sistêmica foi observada em maior proporção quando comparada com o observado por Syme (2007). Por outro lado, Souza et al. (2007) observaram aumento da pressão arterial em um significativo número de animais. Apesar das variações entre estudos; no presente trabalho, a hipertensão arterial em gatos hipertireoideos foi mais frequente que vários outros parâmetros, como a elevação da fosfatase alcalina, por exemplo. Acredita-se que apesar do aumento no débito cardíaco observado em gatos com hipertireoidismo, a redução concomitante da resistência vascular periférica justifique a discreta alteração de pressão nesses animais (Syme, 2007).

\section{CONCLUSÕES}

Todo gato acima de sete anos de idade deve ser avaliado para a presença de lobo tireoidiano cervical palpável e dosagem de tiroxina total sérica.

O tamanho do lobo está relacionado diretamente com o nível sérico de tiroxina total quando o lobo é produtivo. Por isso, quanto mais precocemente o hipertireoidismo for diagnosticado, menor as alterações clínicas e sistêmicas como observado nesse estudo.

Agradecimentos.- À FAPERJ pelo apoio financeiro prestado ao projeto.

\section{REFERÊNCIAS}

Boretti F.S., Sieber-Ruckstuhl N.S., Gerber B., Laluha P., Baumgartner C., Lutz H., Hofmann-Lehmann R. \& Reusch C.E. 2009. Thyroid enlargement and its relationship to clinicopathological parameters and T(4) status in suspected hyperthyroid cats. J. Fel. Med. Surg. 11:286-292.

Broussard J.D., Peterson M.E. \& Fox P.R. 1995. Changes in clinical and laboratory findings in cats with hyperthyroidism from 1983 to 1993. J. Am. Vet. Med. Assoc. 206:302-305.
Camacho A.A. \& Mucha C.J. 2004. Semiologia do Sistema circulatório de Cães e Gatos, p.282-311. In: Feitosa F.L.F. (Ed.), Semiologia Veterinária: a arte do diagnóstico. Roca, São Paulo.

Carlos R.S.A. \& Albuquerque G.R. 2005. Hipertireoidismo felino: relato de caso. Clín. Vet. 57:56-62.

Edney A.T.B. \& Smith P.M. 1986. Study of obesity in dogs visiting veterinary practices in the United Kingdom. Vet. Rec. 118:391-396.

Feldman E.C. \& Nelson R.W. 2004. Canine and Feline Endocrinology and Reproduction. $3^{\text {rd }}$ ed. W.B. Saunders, Missouri, p.152-218.

Gonçalves R.C. 2004. Semiologia do sistema respiratório, p.313-331. In: Feitosa F.L.F. (Ed.), Semiologia Veterinária: arte do diagnóstico. Roca, São Paulo.

Harvey A.M., Hibbert A., Barret E.L., Day M.J., Quiggin A.V., Brannan R.M. \& Caney S.M. 2009. Scintigraphic findings in 120 hyperthyroid cats. J. Fel. Med. Surg. 11:96-106.

Henik R.A. 2002. Ecocardiografia e ultra-som Doppler, p.66-94. In: Tilley L.P. \& Goodwin J. (Eds), Manual de Cardiologia para Cães e Gatos. $3^{a}$ ed. Roca , São Paulo.

Hibbert A.M., Gruffydd-Jones T., Barret E.L., Day M.J. \& Harvey A.M. 2009. Feline thyroid carcinoma: diagnosis and response to high-dose radioactive iodine treatment. J. Fel. Med. Surg. 11:116-124.

Júnior A.R., Haipek K., Oliveira R.A., Daniel A.G.T. \& Taranti L. 2007. Hipertireoidismo em felinos: Revisão de literatura e estudo retrospectivo. Medvep. 5:16-21.

Kass P.H., Peterson M.E., Levy J., James K., Becker D.V. \& Cowgill L.D. 1999. Evaluation of environmental, nutritional and host factors in cats with hyperthyroidism. J. Vet. Intern. Med. 13:323-329.

Meric S.M. 1989. Recognizing the clinical features of feline hyperthyroidism. Vet. Med. 84:956-963.

Naan E.C., Kirpensteijn J., Kooistra H.S. \& Peeters M.E. 2006. Results of thyroidectomy in 101 cats with hyperthyroidism. Vet. Surg. 35:287-293.

Norsworthy G.D., Adams V.J., Mcelhaney M.R. \& Milios J.A. 2002a. Palpable thyroid and parathyroid nodules in asymptomatic cats. J. Fel. Med. Surg. 4:145-151.

Norsworthy G.D., Adams V.J., McElhaney M.R. \& Milios J.A. 2002b. Relationship between semi-quantitative thyroid palpation and total thyroxine concentration in cats with and without hyperthyroidism. J. Fel. Med. Surg. 4:139-143.

Paepe D., Smets P., Van Hoek I., Saunders J., Duchateau L. \& Daminet S. 2008. Within- and between-examiner agreement for two thyroid palpation techniques in healthy and hyperthyroid cats. J. Fel. Med. Surg. 10: 558-565.

Peterson M.E. 2006. Diagnostic tests for hyperthyroidism in cats. Clin. Tech. Small Anim. Pract. 21:2-9.

Peterson M.E. \& Ward C.R. 2007. Etiopathologic findings of hyperthyroidism in cats. Vet. Clin. North Am., Small Anim. Pract. 37:633-645.

Peterson M.E., Kintzer P.P., Cavanagh P.G., Fox P.R., Ferguson D.C., Johnson G.F. \& Becker D.V. 1983. Feline hyperthyroidism: pretreatment clinical and laboratory evaluation of 131 cases. J. Am. Vet. Med. Assoc. 183:103-110.

Richards J.R., Rodan I., Beekman G.K., Carlson M.E., Graves T.K., Kent E.M., Landsberg G.M., Pittari J.M. \& Wolf A.M. 2005. Panel report on Feline Senior Care. American Association of Feline Practitioners/Academy of Feline Medicine. J. Fel. Med. Surg. 7:3-32.

Shiel R.E. \& Mooney C.T. 2007. Testing for hyperthyroidism in cats. Vet. Clin. North Am., Small. Anim. Pract. 37:671-691.

Souza H.J.M., Rezende P.P., Corgozinho K.B., Neves A., Cunha S.C.S. \& Damico C. B. 2007. Estudo preliminar do hipertireoidismo felino: Perfil clínico e laboratorial de 43 casos (1999-2006). Medvep. 5:152-157

Stepien R.L. 2011. Feline systemic hypertension: Diagnosis and management. J. Fel. Med. Surg. 13:35-43.

Syme H.M. 2007. Cardiovascular and renal manifestations of hyperthyroidism. Vet. Clin. North Am., Small Anim. Pract. 37:723-743.

Tilley L.P. 2006. Systemic hypertension, p.144-145. In: Norsworthy G.D., Crystal M.A., Grace S.F. \& Tilley L.P. (Eds), The Feline Patient. $3^{\text {rd }}$ ed. Blackwell Publishing, Iowa. 